\title{
Transcatheter aortic valve implantation after previous mechanical mitral valve replacement in a patient with coexistent rheumatic aortic stenosis and regurgitation
}

\author{
Yuehuan Li ${ }^{1}$, Haibo Zhang ${ }^{1}$, Yujie Zhou ${ }^{2}$, Xu Meng ${ }^{1}$ \\ ${ }^{1}$ Department of Cardiac Surgery, ${ }^{2}$ Vasculocardiology Department, Beijing Anzhen Hospital, Capital Medical University, Beijing 100029, China \\ Correspondence to: Xu Meng, MD. Department of Cardiac Surgery, Beijing Anzhen Hospital Beijing 100029, China. Email: mxu@263.net.
}

\begin{abstract}
A 67-year-old female was referred to our center with rheumatic aortic stenosis (AS) and aortic regurgitation (AI). As the patient was a high-risk case for surgery, we chose transcatheter aortic valve implantation (TAVI) as the treatment of choice. However, the thickened, long, and mildly calcified aortic valve, and the very small distance $(7 \mathrm{~mm})$ between the aortic annulus and mechanical mitral valve (MMV) increased the risk of coronary artery occlusion and flap clamping of MMV. As the left ventricle was small in size, transfemoral TAVI, which causes lesser degree of trauma to the patient, was successfully performed eventually.
\end{abstract}

Keywords: Transcatheter aortic valve implantation (TAVI); rheumatic aortic stenosis (rheumatic AS); mechanical mitral valve (MMV)

Submitted Jun 21, 2019. Accepted for publication Jul 24, 2019.

doi: $10.21037 / \mathrm{cdt} .2019 .07 .14$

View this article at: http://dx.doi.org/10.21037/cdt.2019.07.14

\section{Introduction}

Transcatheter aortic valve implantation (TAVI) is a major method for treatment of high surgical risk patients with aortic stenosis (AS) or patients with AS in whom surgical valve replacement is contraindicated (1). Herein, we report a complicated case where a patient with rheumatic AS and aortic regurgitation (AI) underwent transfemoral TAVI after mechanical mitral valve (MMV) replacement.

\section{Case presentation}

A 67-year-old female was referred to our center with severe rheumatic AS and AI. Previous history revealed hypertension and MMV replacement with a $25 \# \mathrm{On}-\mathrm{X}$ valve (CryoLife, Inc., Kennesaw, GA, USA) 11 years ago. A permanent pacemaker had been implanted 7 years ago for sick sinus syndrome. Transthoracic echocardiography (TTE) revealed severe AS with a flow velocity of $476 \mathrm{~cm} / \mathrm{s}$, mean gradient of $52.6 \mathrm{mmHg}$, combined with moderate tricuspid insufficiency and severe pulmonary hypertension (systolic pulmonary pressure, $89 \mathrm{mmHg}$ ). What's more, the left ventricular end diastolic diameter is only $41 \mathrm{~mm}$ and the left ventricular systolic function is preserved (ejection fraction, 70\%). The coronary and great vessels multi-detector computerized tomography (MDCT) angiography showed no coronary or peripheral artery stenosis. Because the logistic European System for Cardiac Operative Risk Evaluation (EuroSCORE) and Society of Thoracic Surgeons (STS) risk scores (two risk score models, which are used to determine a patient's extent of risk for complications such as mortality after cardiac surgery; they allow physicians to assess a patient's surgical candidacy) were $23.45 \%$ and $8.073 \%$, respectively, TAVI was chosen as the procedure of first choice. However, the long, thickened, mildly calcified aortic valve, and minimal distance $(7 \mathrm{~mm})$ between the aortic annulus and MMV increased the risk of coronary artery occlusion and flap clamping of the MMV. Transapical access might be a feasible alternative method (2). Due to the small size of the left ventricle and the reduced trauma caused by transfemoral TAVI, this procedure was the right choice (Figure 1).

The procedure was performed under local anesthesia 

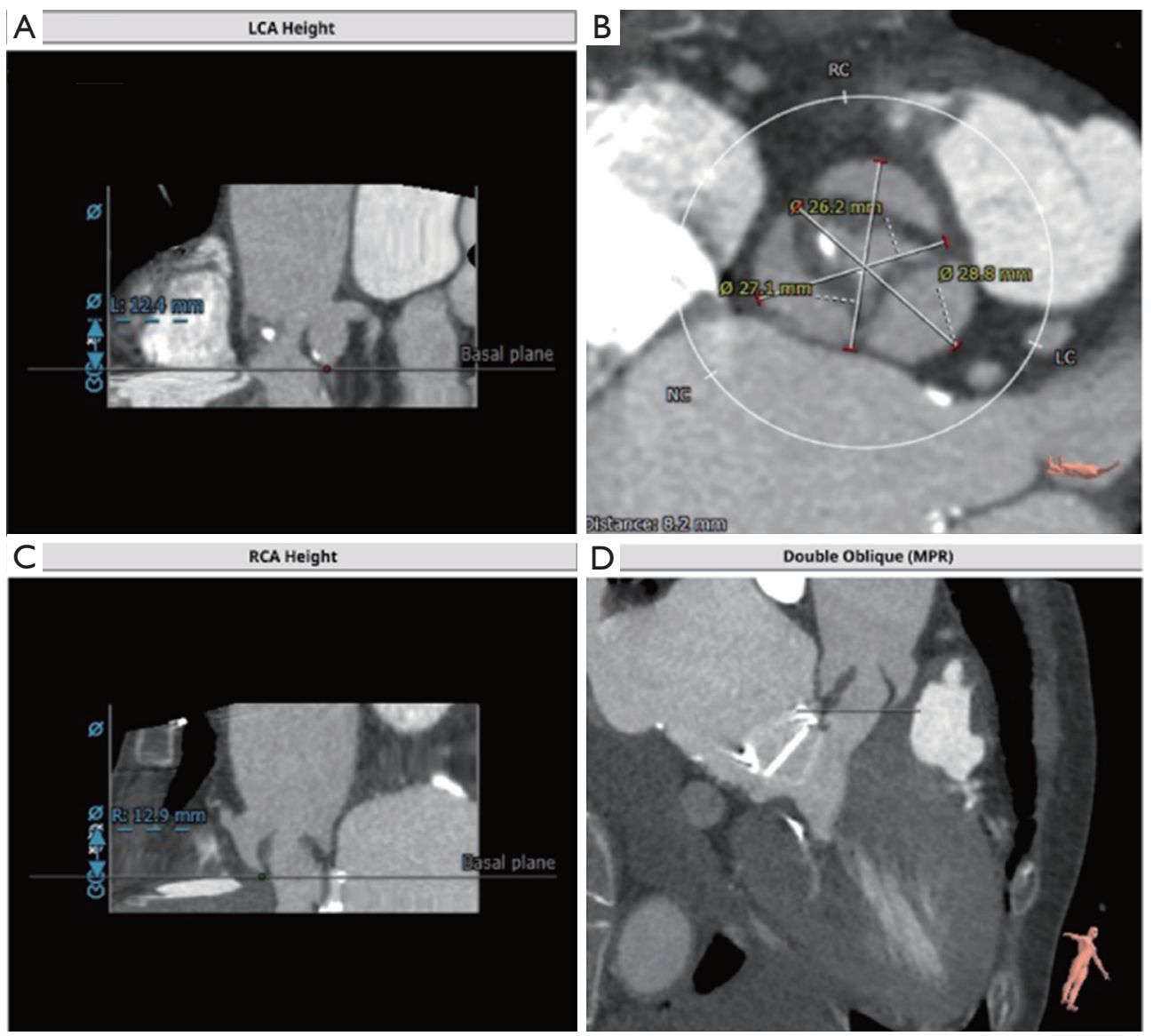

Figure 1 Coronary height is the distance between the origin of the coronary opening and the virtual ring. (A) Left coronary opening height is $12.4 \mathrm{~mm}$; (B) small Valsalva sinus diameters, from 26.2 to $28.8 \mathrm{~mm}$, and mild calcification at the junction of right and non-coronary valve; (C) right coronary opening height is $12.9 \mathrm{~mm}$; (D) very small distance between the aortic annulus and mechanical mitral valve.

with monitored anesthesia care using a self-expandable VenusA-Valve ${ }^{\circledR}$ (VENUSMEDTECH, Hangzhou, China). During the procedure, the aortic valve was butterfingered and sufficiently dilated until the third times using an $18 \mathrm{~mm} \times 40 \mathrm{~mm}$ diameter balloon. Then the $23 \mathrm{~mm}$ valve was delivered and released as usual, keeping the 3 mark points $2-4 \mathrm{~mm}$ above the virtual ring to maintain the best possible coaxiality; the valve was released slowly, allowing the valve to fully adapt to the temperature of the blood and to prevent displacement. When the valve was nearly $1 / 3$ released, and the pacemaker was set to $160 \mathrm{bpm}$, the blood pressure dropped. Focusing on mitral valve movement, we pulled the valve tightly to prevent the valve from slipping into the left ventricular outflow tract. When the valve was $2 / 3$ released, the valve began to work, and the blood pressure rose. After waiting for a moment to confirm that the mechanical valve was working properly and that there was no coronary artery obstruction, the valve was completely released (Figure 2). TTE showed no relevant aortic regurgitation. The maximum velocity $(284 \mathrm{~cm} / \mathrm{s})$ and mean pressure gradient $(16.9 \mathrm{mmHg}$ ) were normal (Figure $3 A$ ). The patient recovered from the procedure without any further complications and was discharged on the $5^{\text {th }}$ postoperative day with a normally functioning aortic biological valve prosthesis. The patient was asymptomatic, with no serious cardiovascular and cerebrovascular adverse events occurred during 6-month follow-up. The prosthesis valve had good form and location demonstrated by MDCT (Figure 3B).

\section{Discussion}

Coronary obstruction is a serious complication of TAVI and the main risk factors include low origin of coronary 

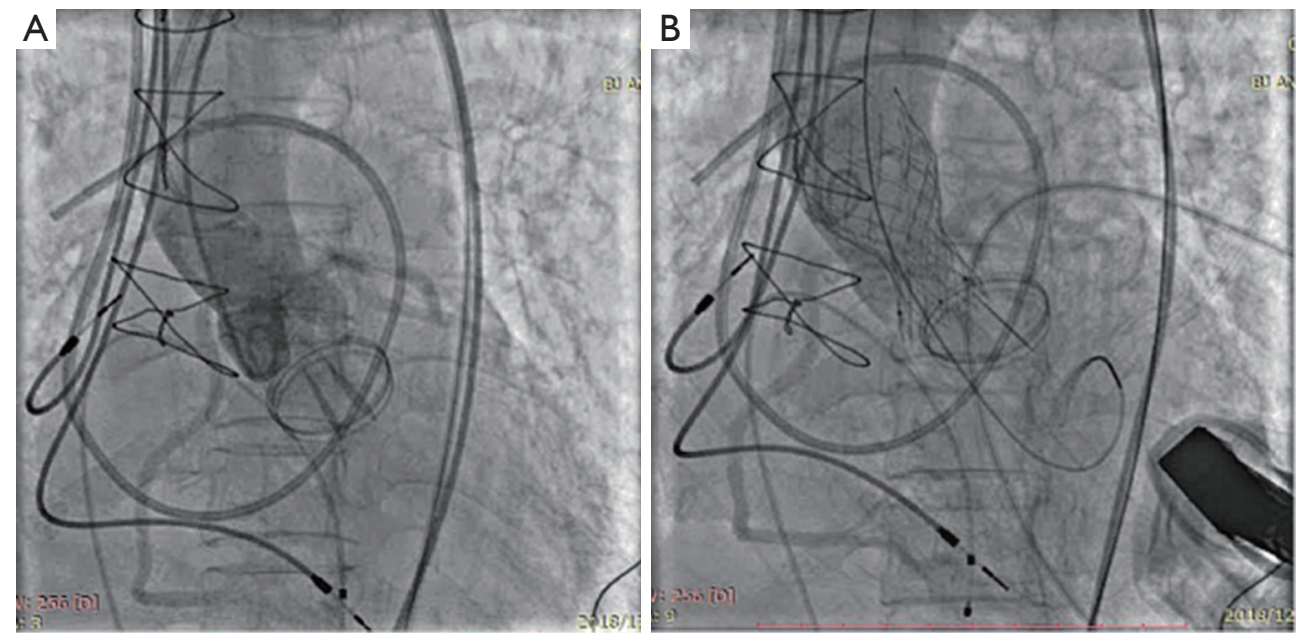

Figure 2 Angiography shows intact coronary artery and mechanical prosthetic valve close to the aortic valve (A); confirmation of proper working of the mechanical valve and absence of coronary artery obstruction after the valve was released (B).
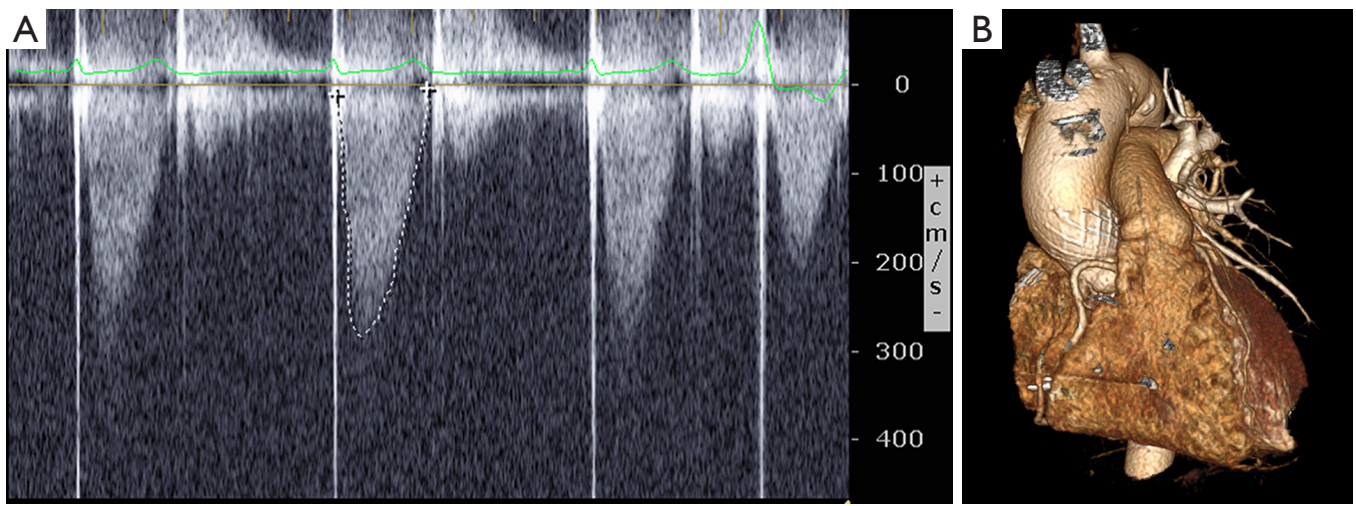

Figure 3 TTE showed the maximum velocity $(284 \mathrm{~cm} / \mathrm{s})$ and mean pressure gradient $(16.9 \mathrm{mmHg})$ were satisfactory (A), without any perivalvular leakage. The prosthesis valve had good form and location demonstrated by MDCT (B). TTE, transthoracic echocardiography; MDCT, multi-detector computerized tomography.

arteries, small sinus of Valsalva, valve misplacement, bulky calcifications, and coronary emboli (3). Moreover, TAVI after previous MMV replacement is considered a high-risk procedure, owing to possible interference with the mitral valve prosthesis (4).

In this case, rheumatic AS and AI with thickened, slipped, and mildly calcified leaves, increased the difficulty of balloon dilation and the risk of valve displacement. Moreover, the very small distance between the prosthesis and aortic ring, was very likely to interfere with the mechanical prosthesis, resulting in a stuck valve. Beller et al. reported five patients with a mean distance of $10 \pm 1 \mathrm{~mm}$ (range, 9-11 $\mathrm{mm}$ ) between the prosthesis and aortic ring in whom TAVI was successfully performed using a balloonexpandable stent (Edwards Lifesciences SAPIEN) (5). What was worse, we had no any balloon-expandable stent. However, the distance was only $7 \mathrm{~mm}$ in our case and the VenusA is longer than the SAPIEN. Furthermore, the aortic valve leaflet was redundant, exceeding the level of the coronary opening. Even more deleterious was the small size of the Valsalva sinus, which greatly increased the risk of coronary artery obstruction. Despite this, the procedure was successfully performed. Higher location, best possible coaxiality, slow release of the valve, advanced pace-making, 
and tightly pulled the valve may be the key factors resulting in successful surgery.

\section{Conclusions}

In conclusion, use of a VenusA prosthesis to treat rheumatic AS combined with AI after previous MMV replacement may be a valid treatment option in such patients.

\section{Acknowledgments}

None.

\section{Footnote}

Conflicts of Interest: The authors have no conflicts of interest to declare.

Ethical Statement: The authors are accountable for all aspects of the work in ensuring that questions related to the accuracy or integrity of any part of the work are appropriately investigated and resolved. Written informed consent was obtained from the patient for publication of this manuscript and any accompanying images.

\section{References}

1. Giannini F, Baldetti L, Gallone G, et al. Transcatheter Valve Replacement in Asia Pacific: Current Practice and Perspectives. J Am Coll Cardiol 2018;72:3189-99.

2. Wachter K, Ahad S, Rustenbach CJ, et al. Transapical aortic valve implantation in patients with pre-existing mitral valve prostheses: a case report. J Cardiothorac Surg 2016;11:133.

3. Dursun H, Gonencer JZ, Karabay O, et al. TAVI in a Patient with Single Coronary Artery: The Choice of Self-Expandable Valve may be Reasonable. Balkan Med J 2016;33:357-9.

4. Gedikli O, Kutlu M, Civelek A, et al. Transcatheter implantation of a CoreValve aortic prosthesis in a patient with a ball-cage mechanical mitral valve. J Heart Valve Dis 2013;22:697-700.

5. Beller CJ, Bekeredjian R, Krumsdorf U, et al. Transcatheter aortic valve implantation after previous mechanical mitral valve replacement: expanding indications? Heart Surg Forum 2011;14:E166-70.
Cite this article as: Li Y, Zhang H, Zhou Y, Meng X. Transcatheter aortic valve implantation after previous mechanical mitral valve replacement in a patient with coexistent rheumatic aortic stenosis and regurgitation. Cardiovasc Diagn Ther 2019;9(5):529-532. doi: 10.21037/cdt.2019.07.14 